\title{
CONTROLE DE PLANTAS DANINHAS TOLERANTES AO GLIFOSATO COM 2,4 D E DICAMBA
}

\author{
Leandro Spíndola Pereira1*; Gustavo Dorneles de Sousa1; Estevam Matheus Costa2; \\ Gustavo Silva de Oliveira1; Matheus Vinicius Abadia Ventura³; Dayana Cardoso Cruz; \\ Adriano jakelaitis ${ }^{4}$
}
${ }^{1}$ Engenheiro Agrônomo, Instituto Federal Goiano (IF Goiano) - campus Rio Verde, Rua do Pequi, 917-1047 - Lot. Gameleira, Rio Verde - GO, CEP: 75906-750
${ }^{2}$ Engenheiro Agrônomo, Mestre em Ciências agrárias, Instituto Federal Goiano (IF Goiano) - campus Rio Verde, Rio Verde - GO ${ }^{3}$ Engenheiro Agrônomo, doutorando em Ciências Agrárias, Instituto Federal Goiano (IF Goiano) - campus Rio Verde, Rio Verde - GO ${ }^{4}$ Professor Doutor, em Fitotecnia, Instituto Federal Goiano (IF Goiano) - campus Rio Verde, Rio Verde - GO
* Autor para correspondência: Leandro Spíndola Pereira, leandrop629@gmail.com

RESUMO: Objetivou-se avaliar os efeitos dos herbicidas dicamba e 2,4 D isolados ou em mistura com glifosato sobre as plantas daninhas tolerantes ao glifosato: apaga-fogo (Alternantera tenella) e agriãozinho (Synedrellopsis grisebachii). Os experimentos foram conduzidos em vasos em casa de vegetação climatizada no Instituto Federal Goiano campus Rio Verde. Foram conduzidos dois ensaios, um para cada espécie, em delineamento em blocos casualizados, com quatro repetições. Os tratamentos foram dispostos em esquema fatorial $11 \times 2$, com quatro repetições, testando onze doses dos herbicidas dicamba e 2,4 D (0; 201,6; 403,2; 694,$8 ; 806,4$ e 1,008 g e.a. ha-1 cada) (fator A) combinados com a presença e ausência do herbicida glifosato (960 g e.a ha-1) (fator B). Foram realizadas avaliações de fitointoxicação aos 7, 14, 21 e 28 dias após a aplicação do herbicida (DAA), e aos 28 DAA as plantas foram cortadas no nível do solo, e transferidas a estufa de renovação e circulação do ar para determinação da biomassa seca. As espécies $S$. grisebachii e $A$. tenella são tolerantes ao herbicida glifosato na dose de $960 \mathrm{~g}$ e.a ha-1. A aplicação dos herbicidas 2,4 D e dicamba, resultam em excelente controle de $A$. tenella, já para $S$. grisebachii foi a aplicação de 2,4 D que resultou em maior controle do que doses de dicamba de até 806,4 g i.a. ha-1.

PALAVRAS-CHAVE: Fitointoxicação; Althernantera tenella; Synedrellopsis grisebachii; mimetizador de auxina.

\section{COTROL OF GLHYPHOSATE TOLERANT WEED WITH 2.4 D AND DICAMBA}

ABSTRACT: The objective of this reasearch was to evalue the effects of the herbicides dicamba and 2,4 D isolated or in the mix with glyphosate over the weeds glyphosate-tolerant: Alternantera tenella e Synedrellopsis grisebachii. The experiments were conduct in the nursery pots in climatized greenhouse at Instituto Federal Goiano - Campus Rio Verde. Two trials were conduct, one for each species in a randomized block design, with four replications. The treatments were arrange in factorial scheme $11 \times 2$, with four replications, testing eleven doses of the herbicides dicamba and 2,4 D (0; 201,6; 403,2; 694,8; 806,4 e 1,008 g e.a. ha-1 cada, each) (fator A) combined with the presence and abscence of the glyphosate herbicide ( $960 \mathrm{~g} \mathrm{e.a} \mathrm{ha-1)} \mathrm{(factor}$ B). Phytotoxicity assessments were carry out at 7, 14, 21 and 28 days after application of the herbicide (DAA), and at 28 DAA the plants were cut at ground level, and transferred to a circulation stove to determine biomass dry. The species S. grisebachii and A. tenella are tolerant to the herbicide glyphosate at the dose of $960 \mathrm{~g}$ e.a ha $^{-1}$. The application of the $2.4 \mathrm{D}$ and dicamba herbicides results in excellent control of $A$. tenella, whereas for S. grisebachii it was the application of $2.4 \mathrm{D}$ that resulted in greater control than doses of dicamba of up to $806.4 \mathrm{~g}_{\text {ia ha }}{ }^{-1}$.

KEY WORDS: Phytointoxication; Althernantera tenella; Synedrellopsis grisebachii; auxin mimic.

\section{INTRODUÇÃO}

Devido ao surgimento de plantas daninhas resistentes e tolerantes ao glifosato, as empresas, buscaram a partir do melhoramento genético de plantas, o desenvolvimento de variedades e cultivares que sejam resistentes a mais de um herbicida (OSIPE et al., 2016), como por exemplo, a soja resistente tanto ao glifosato quanto ao dicamba (tecnologia Intacta 2 Xtende), e a soja resistente ao glifosato e ao 2,4 D Colex (tecnologia Enlist ${ }^{\mathrm{TM}}$ ).

Os herbicidas dicamba e $2,4 \quad D$, são classificados como mimetizadores de auxina, sendo amplamente utilizados e indicados para o controle de plantas daninhas, principalmente dicotiledôneas, em pós emergência das culturas do milho, arroz de sequeiro, trigo, pastagens e outras e/ou em dessecação, visando o controle de plantas daninhas 
resistentes e tolerantes ao glifosato (VIEIRA et al., 1999), podendo ser aplicados, em mistura, com o glifosato ou de forma individual (SOARES et al., 2012).

Estudos apontam que os herbicidas mimetizadores de auxinas, quando aplicados em mistura com o glifosato, podem reduzir a taxa limiar de fitotoxidade do glifosato nas gramíneas (antagonismo), no entanto o aumento das taxas de glifosato, acima do nível limite, superam o antagonismo (SOARES et al., 2012; O'SULLIVAN \& O'DONOVAN, 1980).

No Brasil, as espécies de plantas daninhas identificadas como tolerantes ao glifosato são: ervaquente (Spermacoce latifolia), trapoeraba (Commelina benghalensis), erva-de-touro (Tridax procumbens), trevo mexicano (Richardia brasiliensis), corda de viola (Ipomoea grandifolia), apaga-fogo (Alternantera tenella), agriãozinho (Synedrellopsis grisebachii), e erva-de-santa-luzia (Chamaesyce hirta) (PROCÓPIO et al., 2007; TAKANO et al., 2013; GIANCOTTI et al., 2012; PEREIRA et al., 2011; FOLONI et al., 2005).

Takano et al (2013), trabalhando com o efeito da adição de 2,4 D ao glifosato em plantas daninhas de folhas largas, verificou o aumento nos níveis de controle. Embora o dicamba seja muito utilizado nas culturas de milho e trigo (BEHRENS et al., 2007), tem-se pouca informação relacionada à eficácia do herbicida nessas espécies de plantas daninhas tolerantes, sendo que atualmente, nenhum produto comercial que apresenta como princípio ativo do dicamba é registrado no Brasil (OSIPE et al., 2016).

Avaliar e comparar o efeito dos herbicidas dicamba e 2,4 D, sobre o controle e a redução da massa seca das plantas daninhas tolerantes ao glifosato: apaga-fogo (Alternantera tenella) e agriãozinho (Synedrellopsis grisebachii).

\section{MATERIAL E MÉTODOS}

O experimento foi conduzido em vasos na casa de vegetação climatizada do Instituto Federal Goiano campus Rio Verde, no município de Rio Verde, em Goiás. Dois ensaios foram conduzidos envolvendo as espécies: apaga-fogo (Alternanthera tenella) e agriãozinho (Synedrellopsis grisebachii), que foram cultivadas em solo de textura argiloso, obtido no município de Rio Verde, Goiás. Os solos foram analisados física e quimicamente, sendo necessário a correção ( $\mathrm{V}=60 \%$ ) com calcário filler (PRNT - 92,25\%) antes do transplante das espécies, realizou a adubação química de base dos vasos foi realizado a fim de permitir o desenvolvimento das espécies, aplicando $50 \mathrm{Kg}$ de $\mathrm{N}, 100 \mathrm{Kg}$ de $\mathrm{P}_{2} \mathrm{O}_{5}$ e 50
$\mathrm{Kg}$ de $\mathrm{K}_{2} \mathrm{O}$ ha $^{-1}$, aplicados na forma de sulfato de amônio, termofosfato yorin e cloreto de potássio, respectivamente.

Plântulas contendo dois pares de folhas expandida das respectivas plantas daninhas infestantes foram obtidas em áreas agrícolas, de ocorrência natural, e transplantadas uma planta diretamente por vaso. Cada unidade experimental foi constituída de vasos de polietileno, com a capacidade de $6 \mathrm{dm}^{3}$, contendo $5,5 \mathrm{~kg}$ de solo e uma planta de Alternantera tenella e Synedrellopsis grisebachii. Os vasos foram irrigados sempre que necessários, a fim de manter umidade do solo constante. Sendo que até os 7 dias após a aplicação a irrigação foi realizada de modo a não permitir molhamento foliar.

$O$ delineamento experimental utilizado foi o de blocos ao acaso, disposto em esquema fatorial $11 \mathrm{x}$ 2, com quatro repetições, sendo 11 amostras controles, com e sem a adição do herbicida glifosato (960 g e.a ha-1), totalizando 22 tratamentos. As 11 amostras controles, foram representados pelas doses: 201,$6 ; 403,2 ; 694,8 ; 806,4$ e 1,008 g e.a. ha-1 de dicamba e 2,4 D, mais um tratamento sem aplicação do mimetizador de auxina.

A pulverização dos herbicidas foi realizada quando as plantas daninhas se situavam em florescimento pleno, aos 30 dias após o florescimento apresentaram 6 folhas expandidas ( $A$. tenella) (Figura 1A) e comprimento de plântula de 15 centímetros (Synedrellopsis grisebachii) (Figura $1 \mathrm{~B}$ ). A aplicação foi realizada com auxílio de pulverizador costal pressurizado por cilindro de $\mathrm{CO}_{2}$, equipado com barra de quatro pontas TT 11002, espaçadas de $0,50 \mathrm{~m}$, posicionados a $0,5 \mathrm{~m}$ de altura em relação à superfície das plantas, volume de calda de $200 \mathrm{~L}$ ha1 e pressão de trabalho de 2,5 bar. No momento das aplicações a temperatura do ar situou-se entre 25,0 a $28,0^{\circ} \mathrm{C}$; umidade relativa do ar entre 65,0 a $73,0 \%$,

Realizou as avaliações de controle, fitointoxicação, aos 7, 14, 21, 28 dias após a aplicação do herbicida (DAA), através de uma escala visual, realizada por três avaliadores, de $0-100 \%$, onde $0 \%$ representa efeito nulo do herbicida nas plantas, e $100 \%$ representa efeito total do herbicida na planta (morte). A partir dos sintomas relacionados aos mimetizadores de auxina, baseado na escala de controle e injúria proposta por Frans et al. (1986), que inclui a epinastia e enrolamento de folhas, ramos e pecíolo, e dos sintomas relacionados ao glifosato que inclui clorose seguida a necrose de órgão, como observado nas Figuras 1A, para A. tenella, e 1B, para S. grisebachii, determinou 0 fitointoxicação das espécies infestantes. 
Figura 1. Injurias provocadas pela aplicação dos herbicidas nos ensaios, para as espécies Alternathera tenella (A) e Synedrellopsis grisebachii (B).

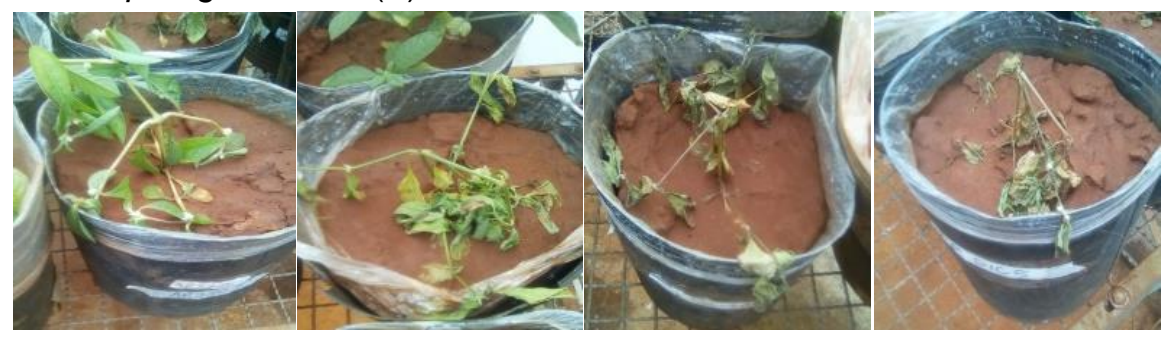

A

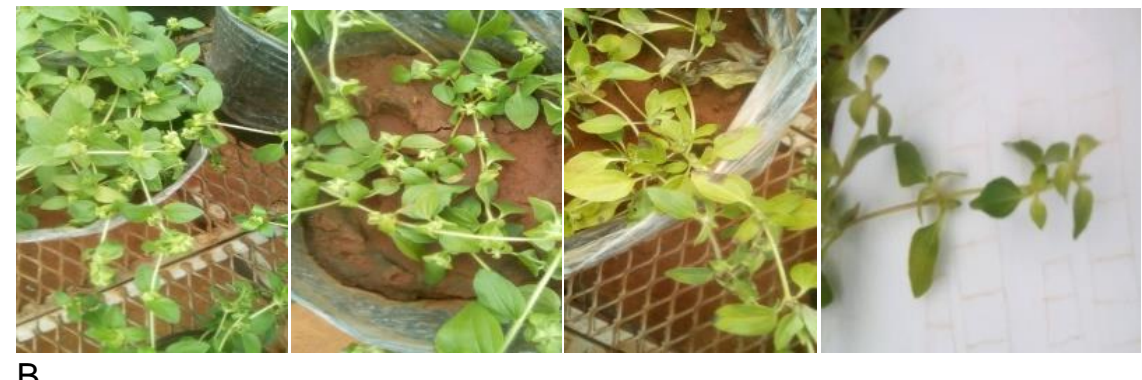

Aos 28 DAA as plantas foram cortadas, e o material foi acondicionado em sacos de papel, e conduzido a estufa de renovação e circulação de ar a $65^{\circ} \mathrm{C}$ por 72 horas, ou até peso constante, a fim de determinar a biomassa seca das plantas.

O conjunto de dados obtidos foram submetidos à análise de variância $(p<0,05)$ e quando significativos as médias foram contrastadas pelo teste Scott Knott a 5\% de probabilidade, por meio do software Sisvar, versão 5.6.

\section{RESULTADOS E DISCUSSÃO}

Para a $A$. tenella, observou interação entre os fatores testados para ambas avaliações de fitointoxicação (7, 14, 21 e 28 DAA) (Tabela 1). Aos 7 DAA observou que a associação dos herbicidas mimetizadores de auxina (dicamba e 2,4 D) ao glifosato resultou efeito dentre as doses aplicadas desses herbicidas, sendo esses sintomas superiores a aplicação isolada de glifosato. Quando realizou a aplicação isolada dos herbicidas dicamba e 2,4 D, observou que doses de dicamba a partir de 403,2 g ha $^{-1}$, resulta em maiores sintomas de fitointoxicação do que doses de dicamba de até $1.008 \mathrm{~g}$, observando também que a associação de glifosato com 2,4 D entre as doses de 201,6 e $1.008 \mathrm{~g}$ e.a. ha-1, e dicamba a dose de 201,6 g e.a. ha-1, resulta em maiores sintomas de injúrias do que as respectivas doses aplicadas isoladamente (Tabela 1).

Aos 14 DAA a associação de dicamba e 2,4 D, independente da dose, com o glifosato, resulta em excelente controle $(>95 \%)$ de $A$. tenella independente da dose, sendo que a associação com 2,4 D nas doses de 201,6 e 403,2 g e.a. ha ${ }^{-1}$ resulta em maiores porcentagens de fitointoxicação do que as respectivas doses do produto aplicado de forma isolada. Aos 14 DAA, menores porcentagens de fitointoxicação foram obtidos para 2,4 D, aplicado isoladamente nas doses de 201,6 e 403,2 g e.a. ha-1 do que a aplicação de dicamba isoladamente a partir da dose de (201,6 g e.a. ha-1) (Tabela 1$)$.

Aos 21 e 28 DAA, observou morte total de $A$. tenella (fitointoxicação - 100\%) para a aplicação de 2,4 D e dicamba isolado ou associado ao glifosato, com exceção da aplicação isolada de 2,4 D na dose de 201,6 g e.a. ha-1 que apresentou fitointoxicação inferior as doses superiores de 2,4 D e doses a partir de 201,6 g e.a ha ${ }^{-1}$ dicamba, e superiores a testemunha sem aplicação, entretanto mesmo esses valores sendo menor, ainda observou um excelente controle $(>90 \%)$ da planta infestante (Frans et al. (1986)).

Vale observar que glifosato aplicado de forma isolada, apresentou menor fitointoxicação do que a aplicação dos herbicidas 2,4 D e dicamba associado ao mesmo, independente da dose e da época de avaliação, não promovendo morte das plantas de $A$. tenella (Tabela 1). Esses resultados se assemelham aos de Takano et al. (2013), que trabalhando com a aplicação de 2,4-D associado ao glyphosate para o controle de espécies de plantas daninhas de difícil controle, observou que a mistura de mimetizador de auxina e glifosato é determinante para acelerar e melhorar o controle de plantas daninhas de difícil controle.

Um dos fatores que podem levar a tolerância de uma espécie ao glyphosate é a alta presença de lignina na superfície da folha o que culmina na menor absorção do mesmo pelas estruturas fotossintéticas, e consequentemente uma dose que controla ampla gama de espécies infestantes não controla uma determinada espécie, como é o caso de $A$. tenella (Carvalho et al., 2009). Na pesquisa foi observado que glyphosate aplicado de forma isolada nas doses de até $960 \mathrm{~g}$ e.a ha-1 controlam A. tenella, causando somente sintomas leves de injúrias (Tabela 1). 
Tabela 1. Avaliações de fitointoxicação de Althernantera tenella aos 7, 14, 21 e 28 DAA de Dicamba e 2,4 D.

\begin{tabular}{|c|c|c|c|c|c|c|c|}
\hline \multicolumn{8}{|c|}{ Althernantera tenella } \\
\hline \multirow{3}{*}{$\begin{array}{l}\text { Herbicidas } \\
\text { Auxinícos }\end{array}$} & \multirow{3}{*}{$\begin{array}{c}\text { Dose } \\
\text { (g i.a. ha- } \\
1 \text { ) }\end{array}$} & \multicolumn{3}{|c|}{ Fitointoxicação aos 7 DAA (\%) } & \multicolumn{3}{|c|}{ Fitointoxicação aos 14 DAA (\%) } \\
\hline & & \multicolumn{2}{|c|}{ Glifosato (960 g e.a ha- } & \multirow{2}{*}{ Médias } & \multicolumn{2}{|c|}{ Glifosato (960 g e.a. ha-1) } & \multirow{2}{*}{ Médias } \\
\hline & & Sem & Com & & Sem & Com & \\
\hline \multirow[t]{3}{*}{ Testemunha } & ------ & $0,0 \mathrm{eA}^{1}$ & $5,4 \mathrm{bA}$ & 2,7 & $0,0 \mathrm{cB}$ & $6,75 \mathrm{bA}$ & 3,3 \\
\hline & 201,6 & $66,7 \mathrm{cB}$ & $91,1 \mathrm{aA}$ & 78,9 & 100,0 aA & 100,0 aA & 100,0 \\
\hline & 403,2 & $90,2 \mathrm{aA}$ & 92,5 aA & 91,3 & $100,0 \mathrm{aA}$ & $100,0 \mathrm{aA}$ & 100,0 \\
\hline \multirow[t]{5}{*}{ Dicamba } & 694,8 & 95,5 aA & 93,8 aA & 94,6 & $100,0 \mathrm{aA}$ & $100,0 \mathrm{aA}$ & 100,0 \\
\hline & 806,4 & 95,8 aA & $98,2 \mathrm{aA}$ & 97,3 & $100,0 \mathrm{aA}$ & $100,0 \mathrm{aA}$ & 100,0 \\
\hline & 1.008 & $100,0 \mathrm{aA}$ & $99,8 \mathrm{aA}$ & 99,9 & $100,0 \mathrm{aA}$ & $100,0 \mathrm{aA}$ & 100,0 \\
\hline & 201,6 & $57,4 \mathrm{~dB}$ & $87,7 \mathrm{aA}$ & 73,0 & $89,1 \quad b B$ & 99,8 aA & 94,4 \\
\hline & 403,2 & $58,1 \mathrm{~dB}$ & 87,1 aA & 73,1 & $89,3 \mathrm{bB}$ & 99,9 aA & 94,6 \\
\hline \multirow[t]{3}{*}{$2,4 \mathrm{D}$} & 694,8 & $72,7 \mathrm{cB}$ & 88,0 aA & 80,3 & $96,1 \mathrm{aA}$ & $100,0 \mathrm{aA}$ & 98,0 \\
\hline & 806,4 & $74,8 \mathrm{cB}$ & $95,1 \mathrm{aA}$ & 85,0 & $100,0 \mathrm{aA}$ & $100,0 \mathrm{aA}$ & 100,0 \\
\hline & 1.008 & $81,0 \mathrm{bB}$ & $98,8 \mathrm{aA}$ & 89,9 & $100,0 \mathrm{aA}$ & $100,0 \mathrm{aA}$ & 100,0 \\
\hline Médias & & 72,0 & 85,4 & & 88,5 & 91,5 & \\
\hline \multirow[t]{2}{*}{ CV (\%) } & \multicolumn{4}{|c|}{8,18} & \multicolumn{3}{|c|}{3,14} \\
\hline & & \multicolumn{3}{|c|}{ Fitointoxicação aos 21 DAA (\%) } & \multicolumn{3}{|c|}{ Fitointoxicação aos 28 DAA (\%) } \\
\hline \multirow[t]{3}{*}{ Testemunha } & ------ & $0,0 \mathrm{cB}$ & $23,5 \mathrm{bA}$ & 11,76 & $0,0 \mathrm{cB}$ & $35,5 \mathrm{bA}$ & 17,7 \\
\hline & 201,6 & $100,0 \mathrm{aA}$ & $100,0 \mathrm{aA}$ & 100,0 & $100,0 \mathrm{aA}$ & $100,0 \mathrm{aA}$ & 100,0 \\
\hline & 403,2 & $100,0 \mathrm{aA}$ & $100,0 \mathrm{aA}$ & 100,0 & $100,0 \mathrm{aA}$ & $100,0 \mathrm{aA}$ & 100,0 \\
\hline \multirow[t]{5}{*}{ Dicamba } & 694,8 & $100,0 \mathrm{aA}$ & $100,0 \mathrm{aA}$ & 100,0 & $100,0 \mathrm{aA}$ & $100,0 \mathrm{aA}$ & 100,0 \\
\hline & 806,4 & $100,0 \mathrm{aA}$ & $100,0 \mathrm{aA}$ & 100,0 & $100,0 \mathrm{aA}$ & $100,0 \mathrm{aA}$ & 100,0 \\
\hline & 1.008 & $100,0 \mathrm{aA}$ & $100,0 \mathrm{aA}$ & 100,0 & $100,0 \mathrm{aA}$ & $100,0 \mathrm{aA}$ & 100,0 \\
\hline & 201,6 & 93,7 bB & $100,0 \mathrm{aA}$ & 95,75 & $94,4 \mathrm{bB}$ & $100,0 \mathrm{aA}$ & 97,2 \\
\hline & 403,2 & 100,0 aA & 100,0 a $A$ & 100,0 & $100,0 \mathrm{aA}$ & $100,0 \mathrm{aA}$ & 100,0 \\
\hline \multirow[t]{3}{*}{$2,4 \mathrm{D}$} & 694,8 & $100,0 \mathrm{aA}$ & $100,0 \mathrm{aA}$ & 100,0 & $100,0 \mathrm{aA}$ & $100,0 \mathrm{aA}$ & 100,0 \\
\hline & 806,4 & $100,0 \mathrm{aA}$ & 100,0 a $A$ & 100,0 & $100,0 \mathrm{aA}$ & $100,0 \mathrm{aA}$ & 100,0 \\
\hline & 1.008 & $100,0 \mathrm{aA}$ & $100,0 \mathrm{aA}$ & 100,0 & $100,0 \mathrm{aA}$ & $100,0 \mathrm{aA}$ & 100,0 \\
\hline Médias & & 90,3 & 93,0 & & 90,4 & 94,1 & \\
\hline
\end{tabular}

${ }^{1}$ Médias seguidas pelas mesmas letras minúsculas nas colunas e maiúsculas na linha são estatisticamente iguais pelo teste de Scott Knott a 5\% de significância.

A mistura em tanque de dois ou mais produtos podem resultar em efeitos aditivos, sinérgicos ou antagônicos. Estudos realizados por Takano et al. (2013), visando avaliação dos efeitos de interação em tanque de 2,4 D e glyphosate, mostraram efeito sinérgico entre as moléculas testadas, dessa forma o melhor controle inicial (7 DAA) de A. tenella, através da associação das moléculas de $2,4 \mathrm{D}$, nas doses testadas, e de dicamba $\left(201,6 \mathrm{~g}^{\text {i.a ha }}{ }^{-1}\right)$ com o glyphosate podem ser resultado de efeito sinérgicos entre os herbicidas mimetizadores de auxina com o inibidor da EPSPs (Tabela 1).

A velocidade de controle e o próprio controle podem mudar em função da espécie de planta daninha, e do produto químico utilizado. A espécie $S$. grisebachii se comportou diferente em relação a $A$. tenella, submetido aos menos produtos químicos. De acordo com a tabela 2, aos 7 DAA, observou interação entre os fatores, não observando diferença entre mistura de produtos e aplicação de 2,4D e dicamba isolados, exceto para maior dose $2,4 \mathrm{D}$ e dicamba (1.008 g e.a ha-1), onde foram observados maiores fitointoxicação com a associação do herbicida glifosato. Aos 7 DAA, a aplicação isolada dos produtos dicamba e 2,4 D, a partir da dose de $806,4 \mathrm{~g}$ e.a ha ${ }^{-1}$ resulta em melhores controles, já quando se associa com o herbicida glifosato, a dose de $1.008 \mathrm{~g}$ e.a. resulta em maiores valores de fitointoxicação. Dessa forma, observa-se resposta de controle inicial de $S$. grisebachii similar entre as aplicações de 2,4 D e dicamba, associado ou não com glifosato (Tabela 2), contrariamente ao observado para $A$. tenella onde melhor efeito de controle incial foi obtido com a aplicação de dicamba isolado em relação ao 2,4 D isolado (Tabela 1).

Aos 14 e 21 DAA observou efeito para os fatores testados de forma isolada, sendo que a adição do glifosato aos herbicidas, resulta em maiores valores de fitointoxicação aos 14 e 21 DAA, bem como a aplicação de dicamba e 2,4 $\mathrm{D}$ na maior dose, $1008 \mathrm{~g}$ e.a. ha-1, aos 14 DAA, e a aplicação de dicamba (1.008 g e.a. ha- $\left.{ }^{-1}\right)$ e 2,4 D (806,4 e $1.008 \mathrm{~g}$ 
e.a. ha-1 $^{-1}$ ) resulta em maiores fitointoxicação, aos 14 associação ou não com o herbicida glifosato (Tabela e 21 DAA, respectivamente, independente da 2).

Tabela 2. Avaliações de fitointoxicação de Synedrellopsis grisebachii aos 7, 14, 21 e 28 DAA de Dicamba e $2,4 \mathrm{D}$.

\begin{tabular}{|c|c|c|c|c|c|c|c|}
\hline \multicolumn{8}{|c|}{ Synedrellopsis grisebachii } \\
\hline \multirow{3}{*}{$\begin{array}{l}\text { Herbicidas } \\
\text { Auxinícos }\end{array}$} & \multirow{3}{*}{$\begin{array}{c}\text { Dose } \\
(\text { g i.a. ha-1) }\end{array}$} & \multicolumn{3}{|c|}{ Fitointoxicação aos 7 DAA (\%) } & \multicolumn{3}{|c|}{ Fitointoxicação aos 14 DAA (\%) } \\
\hline & & \multicolumn{2}{|c|}{ Glifosato (960 g e.a. ha-1) } & \multirow{2}{*}{ Médias } & \multicolumn{2}{|c|}{ Glifosato $(960 \mathrm{~g}$ e.a. ha-1) } & \multirow{2}{*}{ Médias } \\
\hline & & Sem & Com & & Sem & Com & \\
\hline \multirow[t]{3}{*}{ Testemunha } & ------ & $0,0 \mathrm{dA}^{1}$ & $4,6 \mathrm{eA}$ & 2,3 & 0,0 & 27,8 & $13,9 d$ \\
\hline & 201,6 & $7,6 \mathrm{cA}$ & $9,4 \mathrm{dA}$ & 8,5 & 15,8 & 16,2 & $16,0 d$ \\
\hline & 403,2 & $13,9 \mathrm{bA}$ & $17,3 \mathrm{cA}$ & 15,9 & 22,0 & 49,4 & $35,7 \mathrm{c}$ \\
\hline \multirow[t]{5}{*}{ Dicamba } & 694,8 & $16,3 \mathrm{bA}$ & $20,0 \mathrm{bA}$ & 18,1 & 35,5 & 48,8 & $42,1 \mathrm{~b}$ \\
\hline & 806,4 & $23,2 \mathrm{aA}$ & $23,7 \mathrm{bA}$ & 23,5 & 45,1 & 62,7 & $53,9 \mathrm{~b}$ \\
\hline & 1.008 & $26,5 \mathrm{aB}$ & $32,1 \mathrm{aA}$ & 29,3 & 56,5 & 80,9 & $68,7 \mathrm{a}$ \\
\hline & 201,6 & $7,2 \mathrm{cA}$ & $10,9 \mathrm{dA}$ & 9,0 & 10,9 & 62,8 & $36,8 \mathrm{c}$ \\
\hline & 403,2 & $14,8 \mathrm{bA}$ & $11,5 \mathrm{dA}$ & 13,1 & 24,5 & 65,2 & $44,9 \mathrm{~b}$ \\
\hline \multirow[t]{3}{*}{$2,4 \mathrm{D}$} & 694,8 & $17,3 \mathrm{bA}$ & $16,6 \mathrm{cA}$ & 17,0 & 20,8 & 82,1 & $51,4 \mathrm{~b}$ \\
\hline & 806,4 & $20,5 \mathrm{aA}$ & $21,1 \mathrm{bA}$ & 20,8 & 39,6 & 74,3 & $56,9 \mathrm{~b}$ \\
\hline & 1.008 & $22,2 \mathrm{aB}$ & $32,2 \mathrm{aA}$ & 27,2 & 46,9 & 98,0 & $72,4 \mathrm{a}$ \\
\hline Médias & & 15,4 & 18,1 & & $28,9 \mathrm{~B}$ & $60,7 \mathrm{~A}$ & \\
\hline \multirow[t]{2}{*}{ CV (\%) } & \multicolumn{4}{|c|}{19,37} & \multicolumn{3}{|c|}{21,26} \\
\hline & & \multicolumn{3}{|c|}{ Fitointoxicação aos 21 DAA } & \multicolumn{3}{|c|}{ Fitointoxicação aos 28 DAA } \\
\hline \multirow[t]{3}{*}{ Testemunha } & $\begin{array}{c}------ \\
\end{array}$ & 0,0 & 28,7 & $14,6 \mathrm{e}$ & $0,0 \mathrm{~dB}$ & $28,7 \mathrm{cA}$ & 14,3 \\
\hline & 201,6 & 28,7 & 29,3 & $28,7 d$ & 25,4 & $26,1 \mathrm{cA}$ & 25,7 \\
\hline & 403,2 & 55,4 & 78,7 & $67,0 \mathrm{c}$ & 65,4 & 86,6 bA & 76,0 \\
\hline \multirow[t]{5}{*}{ Dicamba } & 694,8 & 72,1 & 89,9 & $81,0 \mathrm{~b}$ & 68,1 & $87,3 \mathrm{bA}$ & 77,7 \\
\hline & 806,4 & 80,3 & 86,4 & $83,3 \mathrm{~b}$ & 89,2 & $85,4 \mathrm{bA}$ & 87,3 \\
\hline & 1.008 & 83,5 & 97,4 & 90,5 a & 91,3 & $97,3 \mathrm{aA}$ & 94,3 \\
\hline & 201,6 & 70,6 & 97,4 & $84,0 \mathrm{~b}$ & 70,2 & $100,00 \mathrm{aA}$ & 85,1 \\
\hline & 403,2 & 75,6 & 90,7 & 82,8 b & 75,4 & $100,00 \mathrm{aA}$ & 87,7 \\
\hline \multirow[t]{3}{*}{$2,4 \mathrm{D}$} & 694,8 & 67,7 & 97,1 & 82,4 b & 76,4 & $100,00 \mathrm{aA}$ & 88,2 \\
\hline & 806,4 & 93,2 & 98,3 & $95,7 \mathrm{a}$ & 93,7 & $100,00 \mathrm{aA}$ & 96,8 \\
\hline & 1.008 & 94,5 & 99,7 & $97,1 \mathrm{a}$ & 98,3 & $100,00 \mathrm{aA}$ & 99,1 \\
\hline Médias & & $65,6 \mathrm{~B}$ & $81,2 \mathrm{~A}$ & & 68,5 & 82,8 & \\
\hline
\end{tabular}

${ }^{1}$ Médias seguidas pelas mesmas letras minúsculas nas colunas e maiúsculas na linha são estatisticamente iguais pelo teste de Scott Knott a $5 \%$ de significância.

Aos 28 DAA a emergência, observou interação entre os fatores. A associação dos herbicidas dicamba e 2,4 $\mathrm{D}$ com o glifosato, resulta em maiores valores de fitointoxicação para o 2,4 D, independente da dose testada, e dicamba na dose de $1.008 \mathrm{~g}$ e.a. $\mathrm{ha}^{-1}$. A aplicação isolada dos herbicidas resulta em maiores valores de fitointoxicação para a aplicação de dicamba e 2,4 D a partir da dose de 806,4 g e.a. ha $^{-1}$ (Tabela 2). De forma similar aos resultados obtidos para $A$. tenella, a espécie $S$. grisebachii, também se mostrou tolerante ao herbicida, visto que a aplicação isolada de glifosato na dose de $960 \mathrm{~g}$ e.a. ha $^{-1}$ não resultou em morte da espécie infestante. Também foi possível observar para $S$. grisebachii, uma menor eficiência do produto dicamba em relação ao 2,4 D, quando associados com glifosato, já quando os mesmos são aplicados de forma isolado a eficiência de controle não se diferencia, mostrando que para o controle dessa espécie infestante, pensando em associação ao glifosato a utilização do herbicida 2,4 D, poderá ser mais vantajosa. Os resultados obtidos nessa pesquisa se assemelham aos obtidos por Osipe et al. (2016), onde trabalhando com a aplicação de 2,4 D e dicamba associado ao não com glifosato, para o controle de Commelina benghalensis, Richardia brasiliensis, Conyza sp., e Ipomea nil. observaram que a associação de glifosato aos herbicidas auxínicos contribuiu para a redução da diferença de controle entre as espécies quando os mesmos são aplicados de formas isoladas, o que pode ser resultado de efeito aditivo ou sinérgico entre as moléculas, mostrando também que quando se compara doses equivalentes de 2,4 D 
e dicamba as espécies infestantes podem apresentar efeito diferenciado de controle.

Para a massa seca da parte aérea, observou efeito isolado de ambos fatores para a espécies $A$. tenella, observando que a associação de glifosato a herbicidas e a aplicação isolada (testemunha com glifosato), resulta em redução da matéria seca independente do herbicida auxinícos e da dose, bem como, a aplicação de dicamba e 2,4 a partir da dose de 201,6 (g i.a. ha-1), resulta em maiores valores do que a testemunha, independente da aplicação associado ao não com o glifosato (Tabela 3 ).

Tabela 3. Massa seca da parte aérea (MSPA) de Althernantera tenella e Synedrellopsis grisebachii.

\begin{tabular}{|c|c|c|c|c|}
\hline \multicolumn{5}{|c|}{ Althernantera tenella } \\
\hline \multirow{3}{*}{ Herbicidas Auxinícos } & \multirow{3}{*}{$\begin{array}{c}\text { Dose } \\
(\text { g i.a. ha-1) }\end{array}$} & \multicolumn{3}{|c|}{ Massa seca da parte aérea por planta } \\
\hline & & \multicolumn{2}{|c|}{ Glifosato (960 g e.a. ha-1) } & \multirow{2}{*}{ Médias } \\
\hline & & Sem & Com & \\
\hline \multirow[t]{3}{*}{ Testemunha } & ------- & 5,2836 & 5,3099 & $5,2967 b^{1}$ \\
\hline & 201,6 & 2,0160 & 1,6171 & $1,8166 \mathrm{a}$ \\
\hline & 403,2 & 1,8697 & 1,5569 & $1,7132 \mathrm{a}$ \\
\hline \multirow[t]{5}{*}{ Dicamba } & 694,8 & 1,3694 & 1,1536 & $1,2615 a$ \\
\hline & 806,4 & 1,4985 & 0,8443 & $1,1714 \mathrm{a}$ \\
\hline & 1.008 & 1,6593 & 1,0523 & $1,3558 \mathrm{a}$ \\
\hline & 201,6 & 1,5960 & 1,0648 & $1,3304 \mathrm{a}$ \\
\hline & 403,2 & 1,8101 & 0,8703 & $1,3402 \mathrm{a}$ \\
\hline \multirow[t]{3}{*}{$2,4 \mathrm{D}$} & 694,8 & 1,4346 & 0,9624 & $1,1985 a$ \\
\hline & 806,4 & 1,6707 & 0,9410 & $1,3058 \mathrm{a}$ \\
\hline & 1.008 & 1,5215 & 1,0255 & $1,2735 a$ \\
\hline \multicolumn{2}{|l|}{ Médias } & $1,9754 \mathrm{~B}$ & $1,4907 \mathrm{~A}$ & \\
\hline CV (\%) & \multicolumn{4}{|c|}{19,73} \\
\hline \multicolumn{5}{|c|}{ Synedrellopsis grisebachii } \\
\hline \multirow[t]{3}{*}{ Testemunha } & ------ & 7,1582 & 7,5314 & $7,3448 \mathrm{~d}$ \\
\hline & 201,6 & 5,6787 & 6,4961 & $6,0874 \mathrm{c}$ \\
\hline & 403,2 & 4,9805 & 4,0030 & $4,4917 b$ \\
\hline \multirow[t]{3}{*}{ Dicamba } & 694,8 & 4,4456 & 3,7373 & $4,0915 b$ \\
\hline & 806,4 & 3,8981 & 3,3863 & $3,6425 b$ \\
\hline & 1.008 & 3,8946 & 3,9612 & $3,9279 b$ \\
\hline \multirow{5}{*}{$2,4 \mathrm{D}$} & 201,6 & 2,0111 & 1,9892 & $2,0001 \mathrm{a}$ \\
\hline & 403,2 & 2,8900 & 2,8987 & $2,8943 a$ \\
\hline & 694,8 & 2,6633 & 3,1105 & $2,8869 a$ \\
\hline & 806,4 & 2,0681 & 2,7932 & $2,4306 a$ \\
\hline & 1.008 & 2,4883 & 2,7759 & $2,6321 \mathrm{a}$ \\
\hline Médias & & 3,8342 & 3,8802 & \\
\hline
\end{tabular}

${ }^{1}$ Médias seguidas pelas mesmas letras minúsculas nas colunas e maiúsculas na linha são estatisticamente iguais pelo teste de Scott Knott a 5\% de significância.

Para a massa seca da espécie $S$. grisebachii, observou que a aplicação do herbicida $2,4 \mathrm{D}$ nas doses de 201,6; 403,2; 694,8; 806,4 e 1,008 g e.a. ha $^{-1}$ resulta em menores valores para a variável, independente da associação ou não com o herbicida glifosato (Tabela 3), o que está relacionado com maiores valores observados na avaliação de fitointoxicação aos 28 DAA para essas espécies (Tabela 2). Embora não tenha sido observado interação entre os fatores testados para a massa seca das plantas (Tabela 3 ), ressalta-se que em recomendações de controle de $A$. tenella $e S$. grisebachii a associação dos produtos auxinícos com o glifosato deve ser levada em consideração, visto que em determinadas doses observou incremento no controle das plantas infestantes devido a associação dos herbicidas auxíncios com glifosatos (Tabela 1 e 2), além de que condições de estresse hídrico, a falta de água no sistema reduz a translocação e absorção do produtos químicos, resultando em menor eficiência dos produtos utilizados (SILVA et al., 2019).

A associação de glifosato aos herbicidas dicamba e 2,4 D aumentam a intensidade das injúrias em plantas de $A$. tenella, promovendo maior redução de massa seca. A aplicação dos herbicidas 2,4 D e dicamba, independente da associação com o 
herbicida glifosato, resultam em excelente controle de $A$. tenella e redução da matéria seca.

Para a espécie $S$. grisebachii, a associação de glifosato ao herbicida 2,4 D, independente da dose, e ao dicamba $\left(1.008 \mathrm{~g}\right.$ i.a. ha $\left.^{-1}\right)$ resulta em maiores valores de fitointoxicação. Maior redução de massa seca de S. grisebachii foi observada para a aplicação de 2,4 D independente da dose. As espécies infestantes, $S$. grisebachii e $A$. tenella são tolerantes ao herbicida glifosato na dose de $960 \mathrm{~g}$ e.a. ha-1.

\section{REFERÊNCIAS BIBLIOGRÁFICAS}

Behrens, M. R.; Mutlu, N.; Chakraborty, S.; Dumitru, R.; Jiang, W.Z.; Vallee, B. J.; Weeks, D. P. Dicamba resistance: enlarging and preserving biotechnologybased weed management strategies. Science, 2007, 316, 1185-1188.

Carvalho, S. J. P. D.; Damin, V.; Dias, A. C. R.; Melo, M. S. C.; Nicolai, M.; Christoffoleti, P. J. Dessecação de plantas daninhas com glyphosate em mistura com ureia ou sulfato de amônio. Planta Daninha, 2009, 27, 2, 353-361

Ferreira, A. C. B.; Barros, A. C.; Lamas, F. M. Manejo de plantas daninhas na cultura do algodoeiro. Embrapa Algodão. Circular Técnica (INFOTECA-E), 2006, 1-8.

Foloni, L.L.; Rodrigues, D.; Ferreira, F.; Miranda, R.; Ono, E. O. Aplicação de glifosato em pósemergência, em soja transgênica cultivada no cerrado. Revista Brasileira de Herbicidas, 2005, 4, 3, 47-58.

Giancotti, P. R. F.; Alves, P. L. C. A.; Yamauti, M. S.; Barroso, A. A. M. Postemergence control and germination characteristics of Synedrellopsis grisebachii. Planta Daninha. 2012, 30, 2, 335-340.

Heap, I. Herbicide resistant weeds. In: Integrated pest management. Springer, Dordrecht. 2014, 281301.

O'Sullivan, P. A.; O'Donovan, J. T. Interaction between glyphosate and various herbicides for broadleaved weed control. Weed Res, 1980, 20, 4, 255-260.

Osipe, J. B.; Oliveira Jr, R.S.; Constantin, J.; Takano, H. K.; Biffe, D. F. Spectrum of weed control with 2, 4$\mathrm{d}$ and dicamba herbicides associated to glyphosate or not. Planta Daninha, 2017, 35, e017160815.

Pereira, R. A.; Alves, P. L.; Corrêa, M. P.; Dias, T. C. D. S. Influência da cobertura de aveia-preta e milheto sobre comunidade de plantas daninhas e produção de soja. Revista Brasileira de Ciências Agrárias, 2011, 6, 1, 1-10.

Silva, J. N.; Pereira, L. S.; Sousa, G. D.; Oliveira, G. S.; Jakelaitis, A. Coexistence of soybean plants and Urochloa spp. under glyphosate and water deficit effects. Científica, 2019, 47, 1, 36-45.

Soares, D. J.; Oliveira, W. S.; López-Ovejero, R. F.; Christoffoleti, P. J. Control of glyphosate resistant hairy fleabane (Conyza bonariensis) with dicamba and 2, 4-D. Planta Daninha, 2012, 30, 2, 401-406.

Streibig, J. C. Herbicide bioassay. Weed Res. 1988, 28, 479-563.

Takano, H. K.; Junior, R. S. O.; Constantin, J.; Biffe, D. F.; Franchini, L.H.M.; Braz, G. B. P.; Gemelli, A. Efeito da adição do 2,4-D ao glyphosate para o controle de espécies de plantas daninhas de difícil controle. Revista Brasileira de Herbicidas, 2013, 11, 1-14.

Vieira, E. M.; Prado, A. D.; Landgraf, M. D.; Rezende, M. D. O Estudo da adsorção/dessorção do ácido 2, 4 diclorofenoxiacético (2, 4-D) em solo na ausência e presença de matéria orgânica. Química Nova, 1999, 22, 3, 305-308. 